expected, as it induces a myopia where this has not existed before. Myopia is the result, not the cause of the progression.

$\mathrm{X}$. Cases are quoted to illustrate how hypermetropia that has been stationary for years suddenly diminishes, without going on to myopia, because of the presence of a sufficient hypermetropic reserve.

XI. Of the behaviour of this disorder which induces elongation of the globe, it is pointed out that it has an evolutionary course, beginning at some period of childhood, and persisting through adolescence (progressive myopia), though it may become arrested at an earlier age (stationary myopia in children), or go on through adult life (malignant myopia). (The usual age for the onset of this disorder is probably somewhere about twelve to fourteen years).

XII. A passing suggestion as to the nature of this disorder is made.

XIII. During the school period the growth of the eye has but little effect on its refractive condition.

In conclusion, I wish to express my indebtedness to Mr. T. W. Letchworth. Apart from making this work possible through his permission to use the material discussed in this paper, he has helped the writer to get a clear perception of many of the ideas expressed, of which not a few represent what he has been teaching for some years.

To Mr. A. H. Levy I am obliged for permission to use case No. 7, in the series of myopic cases ; and it is a pleasure to acknowledge appreciation of his ready help and sympathetic criticism.

\title{
ASEPTIC SEROUS MENINGITIS FOLLOWING INTRA-OCULAR FOREIGN BODY
}

\author{
BY \\ FranK W. LAw, M.A., M.B. \\ SENIOR HOUSE SURGEON, ROYAL LONDON OPHTHALMIC HOSPITAL
}

I BELIEVE the case described below to be of a very rare type, and worthy of report.

E.W.H.N., male, aged 17 years, was admitted to this hospital on September 25, 1928, having been sent here from the Royal Free Hospital. At 11 a.m. on the morning of admission he had been struck in the right eye and face by some steel splinters while at work; no previous eye trouble was reported.

There was a skin abrasion of the right cheek. The right eye was slightly injected and watering; examination revealed a perforating 
wound of the cornea at 8 o'clock, with a large hole in the iris in a corresponding position, the ragged margins of which were all directed forwards, having presumably been swept that way by a gush of vitreous into the anterior chamber. The anterior chamber was of normal depth and contained vitreous; the pupil was atropised and the lens clear. The ophthalmoscope revealed a foreign body in the bottom of the vitreous with some extravasated blood; the optic disc and upper fundus were normal. Tension was normal and vision $6 / 5$ unaided.

The left eye was normal in all respects, with $6 / 5$ vision.

A steel foreign body was removed at once from the right eye with the aid of the Haab and small hand magnets, through a keratome incision.

September 26,1928. Appearances were satisfactory. The cornea was bright, with many folds in Descemet's membrane. Vitreous floaters were present in large numbers. General condition satisfactory. The patient vomited a little food once in the afternoon.

September 27, 1928. The lids were swollen and oedematous, cornea hazy, and conjunctiva chemotic. The globe was immobile, and the appearance was one of an orbital infection. Evisceration was decided upon. Before this was done the patient was found at 3.0 p.m. to be definitely drowsy and complained of headache. Head retraction was present, and Kernig's sign positive R. and L.

The globe was eviscerated that evening; pus was present in the vitreous. A rubber drain was inserted far back into the orbital cavity; no pus was found in the orbit nor did any drain from here at any subsequent time. Lumbar puncture was performed, and the cerebro-spinal fluid was found to be under considerable pressure; 25 c.c. of clear colourless fluid were withdrawn before the pressure was normal. A culture of the aqueous revealed the presence of S. albus only; examination of the cerebro-spinal fluid showed no excess of cellular elements, nor was any micro-organism revealed on examination of culture.

No special treatment was followed beyond the exhibition of calomel, attention to a very dirty mouth, and the application of hot fomentations every two hours to the region of the right eye.

September 28, 1928. General condition, was a little improved, and the patient stated that he felt better, The temperature, which yesterday had reached $101.6^{\circ}$, was $100.4^{\circ}$. There was much swelling and chemosis present.

September 29,1928 . The patient complained of much pain in the socket, and of feeling very drowsy. Pulse 48 , temp. $101.4^{\circ}$; the right side of the face was oedematous. There was marked weakness of the left grip, and the left arm reflexes were exaggerated. Abdominal reflexes exaggerated and equal; knee jerks weak and equal, ankle 
jerks moderate and equal. Extensor plantar response right and left. Head retraction persisted.

Lumbar puncture was again performed, and the withdrawal of about $1 \mathrm{drachm}$ sufficed to reduce the pressure to normal.

September 30,1928 . Temperature had fallen to normal, pulse was 52. The patient was less drowsy; the oedema of the face had subsided, and the swelling of the socket was less.

October 1, 1928. General condition was improved. Temperature normal, pulse 52. The grips were equal in strength, and a bilateral flexor response was present. Intense chemosis still caused prolapse of conjunctiva between the lids.

From the last date there has been a gradual and steady improvement; on the 7th the conjunctiva was able to be replaced and the lids were strapped over it ; there is now-October 16, 1928-very little chemosis. On the 11 th the patient was allowed to get up and complained of no headache as a result; he now feels perfectly well.

The clinical picture was undoubtedly one of meningitis, and the condition must be considered to have been one of aseptic serous meningeal effusion. Comparable, in a sense, are the pleural effusion which may occur in conjunction with a liver abscess, and the ascites with intestinal new growth.

I am indebted to Miss I. C. Mann for permission to publish this case.

\section{UNUSUAL CASE OF MACULAR DEGENERATION}

BY

Frank W. LaW, M.A., M.B.

SENIOR HOUSE SURGEON, ROYAL LONDON OPHTHALMIC HOSPITAL

A.M., widow, aged 46 years, was admitted to this hospital on August 21 , 1928. She complained that, although vision had been good in both eyes without glasses up to six years ago, and with glasses until two years ago, since that time there had been a gradual deterioration in both eyes. No other symptoms were complained of except a very ill-defined and mild ache in the region of the right eye for the last year. She was wearing + 3.0 D.Sph. R. and L.

$$
+0.5 \text { D.Cyl. }-180^{\circ}
$$

On admission, the eyes were normal externally, the pupils active, and the tension normal. The right optic disc was normal; in the region of the macula there was vaguely defined a circle, of diameter about 1.5 times that of the disc; the circle could with difficulty be 